\section{Differenz, kritische}

G. Schumann

Hannover, Deutschland

\section{Englischer Begriff critical difference}

Definition Differenz zwischen 2 Quantitäten (quantitativen Untersuchungsergebnissen), die sich gerade noch statistisch signifikant unterscheiden.

Beschreibung Zwei quantitative Untersuchungsergebnisse sind unter analytischen Gesichtspunkten signifikant verschieden, wenn der Absolutbetrag ihrer Differenz größer als die kritische Differenz $D_{k}$ ist (Stamm 1982; Costongs et al. 1985):

$$
\mathrm{D}_{\mathrm{k}}=1,96 \times 2^{1 / 2} \times \mathrm{VK}_{\mathrm{a}}=2,77 \times \mathrm{VK}_{\mathrm{a}},
$$

wobei $\mathrm{VK}_{\mathrm{a}}$ die relative Standardabweichung als Maß für die analytische Ungenauigkeit von Tag zu Tag von Kontrollproben im Rahmen der üblichen Qualitätssicherung bedeutet. Wenn die Ergebnisse aus einer Serie stammen, muss der in einer Serie ermittelte VK verwendet werden. Wenn die Differenz zwischen den beiden Ergebnissen $\leq$ die kritische Dif- ferenz ist, reflektiert der numerische Unterschied wahrscheinlich lediglich die Ungenauigkeit des Verfahrens. Die kritische Differenz hat sich für die Longitudinalbeurteilung von 2 quantitativen Untersuchungsergebnissen bewährt. Wenn die intraindividuelle Komponente der biologischen Streuung $\mathrm{VK}_{\mathrm{i}}$ berücksichtigt werden soll (insbesondere bei größeren Zeitintervallen), muss die Gleichung für $D_{k}$ erweitert werden (Ricos et al. 2004):

$$
\mathrm{D}_{\mathrm{k}}=2,77 \times\left(\mathrm{VK}^{2}{ }_{\mathrm{a}}+\mathrm{VK}^{2}{ }_{\mathrm{i}}\right)^{1 / 2}
$$

Bei Ricos et al. (2004) wurde die kritische Differenz (auch als ,reference change value“ bezeichnet) für 261 Messgrößen zusammengestellt.

\section{Literatur}

Costongs GMPJ, Janson PCW, Bas BM et al (1985) Short-term and longterm intraindividual variation and critical difference of haematological laboratory parameters. J Clin Chem Clin Biochem 23:69-76

Ricos C, Cava F et al (2004) The reference change value: a proposal to interpret laboratory reports in serial testing based on biological variation. Scand J Clin Lab Invest 64:175-184

Stamm D (1982) A new concept for quality control of clinical laboratory investigations in the light of clinical requirements and based on reference method values. J Clin Chem Clin Biochem 20:817-824 